Annals of International Medical and Dental Research

E-ISSN: 2395-2822 | P-ISSN: 2395-2814

Vol-8, Issue-1 | January-February 2022

DOI: 10.53339 /aimdr.2022.8.1.36

Page no- 274-286 | Section- Research Article (Obstetrics \& Gynaecology)

\title{
Evaluation of Mood Changes Among Peripartum Women at PK DAS Institute of Medical Science, Vaniyamkulam, Palakkad: A Prospective, Observational, Longitudinal Study
}

\author{
Manjunath G N1*, Haritha $\mathrm{C}^{2}$
}

${ }^{1}$ Assistant Professor, Department of Obstetrics \& Gynaecology, PK DAS Institute of Medical Science. Vaniyamkulam, Kerala, India.

Email: drmanjunath77@gmail.com

Orcid ID: 0000-0002-5028-9565

${ }^{2}$ Assistant Professor, PK DAS Institute of Medical Science. Vaniyamkulam, Kerala, India. Email: haritha930@gmail.com

Orcid ID: 0000-0003-2199-2427

*Corresponding author

Received: 14 September 2021

Revised: 18 October 2021

Accepted: 28 October 2021

Published: 22 December 2021

\begin{abstract}
Background: The perinatal period is well established as an increased risk for development of serious mood disorders. Maternal mental health in developing countries gets less than its due attention. The present study was undertaken to evaluate mood changes in Peripartum period in our population and to identify demographic, obstetric, social and psychosocial risk factors associated with Peripartum depression using established scales. Material \& Methods: A prospective, observational, longitudinal study conducted in PK das institute of medical science, vaniyamkulam, with 387 perinatal women for 12 months (February 2018- January 2019). Various scales EPDS (Edinburgh Postnatal Depression Scale), CMSS (Couple Marital Satisfaction Scale, IMS (Index of Marital Satisfaction), LES (Life Event Scale) were studied in Peripartum Period. Results: Among a total of 387 participants about half 189 (48.8\%) were in 19-25 years of Age. Almost 30\% and $40 \%$ had dissatisfied married life as per the CMS and IMS scales respectively. Just above $42 \%$ were screen positive for depression antenatally with EPDS \& 39\% $(n=151)$ in the immediate postpartum period. From these 151 screen positive cases in immediate postpartum period, 138 participants were followed up at 4-6 weeks (13 were lost to follow up) and up to 115 of $138(83.3 \%)$ were screen positive for depression $(\mathrm{N}=387,29.7 \%)$, which was statistically significant $(\mathrm{p}<0.001)$. With EPDS during antenatal period there was no statistically significant relationship of depression with Education $(\mathrm{p}=$ $0.195)$, Occupation $(\mathrm{p}=0.651)$ and pregnancy planned or unplanned $(\mathrm{p}=$ 0.223), whereas, Joint family, participants with dissatisfied marital relationship had increased risk of depression as evidenced by IMS and CMSS $(\mathrm{p}<0.001)$. Participants with a previous male gender baby had less risk of developing depression $(\mathrm{p}<0.001)$ \& participants with previous 2 female children had increased risk of depression $(p<0.001)$. Conclusions: This study highlights importance of screening for maternal mental health problems during Peripartum period. Depression in immediate postpartum period is good predictor for increased risk of depression at 4-6 weeks postpartum.
\end{abstract}

Keywords:- Postpartum Depression, EPDS, Peripartum Stress \& Anxiety, Marital Satisfaction. 
Annals of International Medical and Dental Research E-ISSN: 2395-2822 | P-ISSN: 2395-2814 Vol-8, Issue-1 | January-February 2022 DOI: 10.53339 /aimdr.2022.8.1.36 Page no- 274-286 | Section- Research Article (Obstetrics \& Gynaecology)

\section{INTRODUCTION}

The postnatal period is well established as an increased risk for development of Serious mood disorders. ${ }^{[1,2,3,4]}$ There are three common forms of postpartum affective illness. $\frac{[5,6]}{6}$ The blues (baby blues, maternity blues), postpartum (or postnatal) depression and puerperal (postpartum or postnatal) psychosis each of which differs in its prevalence, clinical presentation, and management. [7,8,9] Many women experience overwhelming emotions such as joy, excitement, happiness, anticipation, fulfilment, at one end of the spectrum as well as anxiety, frustration, confusion, or sadness/guilt at the other end of the spectrum during pregnancy and especially in the postpartum period. the Diagnostic and Statistical Manual of Mental Disorders (DSM) 5th edition (DSM-5) now classify major depressive episodes "with Peripartum onset,"

Encompassing cases with symptom onset during pregnancy or in the 4 weeks following delivery.[10] An estimated one in seven women experiences Peripartum depression. in pregnancy, reproductive hormone levels in a woman's body are 20-30 times greater than normal. At delivery, hormone levels drop abruptly, along with changes in amino acids, neurotransmitters, and thyroid hormones, the sudden drop in oestrogen, progesterone, endorphins, and other hormones may trigger depression pregnancy and especially in the postpartum period. [3]

\section{Objectives}

The study was conducted with the following objectives
- To identify risk factors associated with postnatal depression, if any and associated with other mood changes.

- To assess Prevalence and risk factors for the psychological Morbidities using Edinburgh postnatal depression scale (EPDS), Depression, Anxiety and Stress scale (DASS) and other questionnaire in perinatal women.

\section{MATERIAL AND METHODS}

Study site: The present study was carried out in, PK das institute of medical sciences, a referral care teaching hospital. After the approval by the ethical research committee, a systematic method of sampling was used. Term Pregnant women meeting eligible criteria were consecutively selected.

Period of study: 12 months (February 2018 January 2019)

Sample size: 387.

\section{Methodology of the study}

Preparation of scales to the Malayalam speaking population.

Various rating scales like Edinburgh Postnatal Depression Scale (EPDS),

Depression Anxiety Stress Scale (DASS-21), Life Event Scale (LES), Couple Marital Satisfaction self-report Scale (CMSS) and Index of Marital Satisfaction (IMS) scale were translated to Malayalam with input from staff nurses, collated, and verified with Google translator. However several times, Google Translator was not conveying the precise meaning and intention of the questions. Precise 
Annals of International Medical and Dental Research

E-ISSN: 2395-2822 | P-ISSN: 2395-2814

Vol-8, Issue-1 | January-February 2022

DOI: $10.53339 /$ aimdr.2022.8.1.36

Page no- 274-286 | Section- Research Article (Obstetrics \& Gynaecology)

idiomatic equivalents in the local vernacular were considered as far as possible.

\section{Statistical Method}

The data was collected and entered into MS excel; coding, refining and cleaning of the data was done. Results were explained in terms of descriptive and inferential statistics. Descriptive statistics explained by frequencies, mean, SD, and range of numeric variables and by proportions and percentage for categorical variables, the scores obtained during the antenatal and postnatal period grouped and described using mean score with SD, proportions, percentages. Chi-squared test of independence was used to test the association between the dependant variable postnatal depressive symptoms and each of the socio demographic, maternal, infant, social support and psychiatric health conditions.

\section{RESULTS}

[Table 1] and adjacent [table 2] show that Maximum of the study participants was Educated up to high school (43.9\%).

[Table 3] show that Most of the study participants were homemakers $(87.9 \%)$.

[Table 5] shows that 241 (62.3\%) had Spontaneous Vaginal deliveries (SVD) While LSCS was required in $36.4 \%$.

[Table 6] shows Distribution of study participants based on gender of babies born in this study population. Out of 387 babies, Majority 205 (52.9\%) were male babies while $182(47.1 \%)$ were female babies.

[Table 7] shows the distribution of study population based on maritalDissatisfaction assessed in the Antenatal period. According to CMSS 112 (28.9\%) were not satisfied with their marital life, Whereas 154 (39.8\%) patients were not satisfied according to IMS scale.

[Table 8] shows that before delivery using Edinburgh postnatal depression scale with cut off $>09$ for screen positive depression, 166 (42.9\%) pregnant women were having screen positive for depression. Whereas 221 (57.1\%) were normal. After delivery prevalence of depression decreased to 151 (39\%), and 236 (61\%) were normal.

[Table 9] shows Distribution of study population based on Depression before and after Delivery as Evaluated by DASS scale, Prior to delivery $35.4 \%$ of the study participant did not have any form of depression. However, this proportion decreased to $32.2 \%$ after delivery using the same scale. 11 (2.8\%) patients were severely depressed before delivery which increased to23 (5.9\%). Also, this difference between before and after delivery in proportion of participants with depression was found to be statistically significant $(p<0.001)$.

[Table 10] shows that on evaluation by DASS scale prior to delivery $22.7 \%$ of the study participant did not have any form of anxiety. However this proportion decreased to $16.0 \%$ after delivery using the same scale. However, this difference in proportion of participants with anxiety was not found to be statistically significant $(\mathrm{p}$ value $=0.122)$.

[Table 11] shows that on evaluation by DASS scale prior to delivery 234 (60.5\%) of the study participant did not have any form of stress. However this proportion. Decreased to $56.1 \%$ after delivery using the same scale. Around 134 
Annals of International Medical and Dental Research

E-ISSN: 2395-2822 | P-ISSN: 2395-2814

Vol-8, Issue-1 | January-February 2022

DOI: $10.53339 /$ aimdr.2022.8.1.36

Page no- 274-286 | Section- Research Article (Obstetrics \& Gynaecology)

(34.6\%) patients had mild to moderate stress. However, this difference in proportion of participants with stress was not found to be statistically significant ( $p$ value -0.100$)$.

[Table 12] shows that on evaluation by EPDS scale prior to delivery $40.6 \%$ of the study participant did not have any form of depression. However this proportion decreased to $16.7 \%$ four to six weeks after delivery using the same scale. Also, this difference in proportion of participants with depression was found to be statistically significant ( $\mathrm{p}$ value $<0.001$ ).

Data derived from table 11 after combining normal, mild and moderate Stress scores to be not having clinical Stress and severe and very severe Stress scores to be designated as having Stress) [Table 13] shows Depression, Anxiety \& Stress as measured using DAS scale before delivery out of 387 patients $77(19.9 \%)$ patients had Severe Depression out of which 72 patients also had Severe Anxiety and 5 patients had severe stress and only 2 patients had Depression alone. 156 (40.3\%) had Anxiety out of which 72 patients also had Depression and 2 patients had severe stress and 84 (21.7\%) patients had Anxiety alone.

Same shows that depression, anxiety \& stress immediately after delivery, Out of387, 81 (20.9\%) patients had severe depression and 4 patients had depression only, 77of them had Anxiety also and 2 had severe stress. Whereas $173(44.7 \%)$ patients had Anxiety out of which 77 of them had depression also, 5 of them had severe stress and only 93(24.03\%) patients had Anxiety alone. As comparable to before delivery only 5patients had severe stress in immediate postpartum also.
[Table 14] shows the Prevalence and Incidence of antenatal and postnatal Depression, Anxiety and Stress using DAS scale, Out of 387 study population $77(19.9 \%)$ were having Antenatal depression which increased to $81(20.9 \%)$ in immediate postpartum period, while incidence of Depression was $25(6.45 \%)$. Anxiety was found in $156(40.3 \%)$ in antenatal period and $173(44.7 \%)$ in postpartum period, with incidence of Anxiety being 88 (22.7\%). Interestingly, Severe to very severe Stress was found in very few $5(1.3 \%)$ of the study population in antenatal period and postnatal period $5(1.3 \%)$ also.

[Table15] shows the association of risk factors with depression using EPDS before delivery, Joint family type had increased risk of depression $(p=0.001)$, patients with dissatisfied marital relationship had increased risk of depression as evidenced by IMS and CMSS( $p<0.001$ ), patients with Previous baby male gender had less risk of developing depression $(\mathrm{p}<0.001)$ and Patients with previous 2 female babies had increased risk of depression $(p<0.001)$ whereas there was no difference with previous one female child. Patient with Life event scale showing $80 \%$ illness due to stress had significant risk of depression $(\mathrm{p}<0.001)$. Patient with Education, planned or un planned Pregnancy and occupation of the patient did not have any association.

[Table 16] shows that Marital Dissatisfaction as measured by IMS scale was associated with depression in immediate postpartum period $(p=0.013)$ whereas, those with CMSS was not statistically significant $(\mathrm{p}=0.071)$.Gender of the present child being male was associated with less risk of depression $(\mathrm{p}=0.013)$ However, 
Annals of International Medical and Dental Research E-ISSN: 2395-2822 | P-ISSN: 2395-2814 Vol-8, Issue-1 | January-February 2022 DOI: 10.53339/aimdr.2022.8.1.36 Page no- 274-286 | Section- Research Article (Obstetrics \& Gynaecology)

there was no association between depression during immediate postpartum mode of delivery and education of patient, planned / un planned pregnancy, type of family, life event related stress, occupation, spontaneous /induced labour,maternal sense of control during labour and mode of delivery.

[Table 17] shows relation between depression (EPDS) and certain selected risk factors. Marital dissatisfaction as measured by IMS and CMSS was significantly associated with immediate postnatal depression $(p=0.001)$. Those with present baby being male $(p<0.001)$ and previous baby being male $(p=0.034)$ had statistically significant less risk of developing depression $(\mathrm{p}<0.001)$. Whereas, sex of present baby being female, there was no difference

Noted. Most 121(65.8\%) patients with life event scale showing only $30 \%$ illness did not have depression compared to $63(34.2 \%)$ who had depression which was statistically significant. There were no statistically significant association noted between educations of patient, pregnancy planned / Unplanned, occupation, type of family. Maternal sense of control during labour as measured by LAS, spontaneous / induced labour and mode of delivery also did not show any statistically significant association with immediate post natal depression.

Table 1:Distribution of study participants based on Age $(\mathrm{n}=387)$.

\begin{tabular}{|l|l|l|}
\hline Age (in years) & Frequency & Percent (\%) \\
\hline $19-25$ & 189 & 48.8 \\
\hline $26-30$ & 158 & 40.8 \\
\hline $31-38$ & 40 & 10.3 \\
\hline Total & 387 & 100.0 \\
\hline
\end{tabular}

Table 2: Distribution of study participants based on Educational status ( $\mathrm{n}=387)$

\begin{tabular}{|l|l|l|}
\hline Educational Status & Frequency & Percent \\
\hline Up to High School & 170 & 43.9 \\
\hline Higher Secondary & 148 & 38.2 \\
\hline Graduate & 59 & 15.2 \\
\hline Postgraduate & 10 & 2.6 \\
\hline Total & 387 & 100.0 \\
\hline
\end{tabular}

Table 3: Distribution of study participants based on occupation $(n=387)$

\begin{tabular}{|l|l|l|}
\hline Occupation & Frequency & Percent \\
\hline Unskilled & 1 & 0.3 \\
\hline Semiskilled & 32 & 8.3 \\
\hline Professional & 14 & 3.6 \\
\hline Home Maker & 340 & 87.9 \\
\hline Total & 387 & 100.0 \\
\hline
\end{tabular}

Copyright: $\odot$ The author(s), published in Annals of International Medical and Dental Research, Vol-8, Issue-1. This is an open access article under the Attribution-Non Commercial 2.0 Generic (CC BY-NC 2.0) license. (https://creativecommons.org/licenses/by-nc/2.0/) 
Annals of International Medical and Dental Research

E-ISSN: 2395-2822 | P-ISSN: 2395-2814

Vol-8, Issue-1 | January-February 2022

DOI: 10.53339/aimdr.2022.8.1.36

Page no- 274-286 | Section- Research Article (Obstetrics \& Gynaecology)

Table 4: Distribution of study participants based on type of family $(n=387)$

\begin{tabular}{|l|l|l|}
\hline Type of family & Frequency & Percent \\
\hline Nuclear & 293 & 75.7 \\
\hline Joint & 94 & 24.3 \\
\hline
\end{tabular}

Table 5: Distribution of study participants based on mode of delivery $(n=387)$

\begin{tabular}{|l|l|l|}
\hline Mode of delivery & Frequency & Percent \\
\hline SVD & 241 & 62.3 \\
\hline Assisted/ Instrumental & 5 & 1.3 \\
\hline LSCS & 141 & 36.4 \\
\hline Total & 387 & 100.0 \\
\hline
\end{tabular}

Table 6: Distribution of study participants based on Gender of baby $(n=387)$

\begin{tabular}{|l|l|l|}
\hline Gender of baby & Frequency & Percent \\
\hline Male & 205 & 53.0 \\
\hline Female & 182 & 47.0 \\
\hline Total & 387 & 100.0 \\
\hline
\end{tabular}

Table 7: Distribution of study participants based on presence of marital dissatisfaction evaluated by CMSS and IMS scale $(\mathrm{n}=387)$

\begin{tabular}{|c|c|c|}
\hline Marital & \multicolumn{2}{|c|}{ CMSS (Couple Marital Satisfaction Scale } \\
\hline \multicolumn{3}{|c|}{ Dissatisfaction } \\
\hline & Frequency(n) & Percent (\%) \\
\hline Present & 112 & 28.9 \\
\hline Absent & 275 & 71.1 \\
\hline \multicolumn{3}{|c|}{ IMS (Index of Marital Satisfaction) scale } \\
\hline Present & 154 & 39.8 \\
\hline Absent & 233 & 60.2 \\
\hline
\end{tabular}

Table 8: Comparison of Edinburgh Postnatal Depression Scale (EPDS) scores before and Immediately after delivery.

\begin{tabular}{|l|l|l|}
\hline & Before delivery $\mathbf{n}=\mathbf{3 8 7}$ & After delivery $\mathbf{n}=387$ \\
\hline Cut off score $>\mathbf{9}$ & Frequency (\%) & Frequency $\mathbf{( \% )}$ \\
\hline Possible depression & $166(42.9 \%)$ & $151(39.0 \%)$ \\
\hline Normal & $221(57.1 \%)$ & $236(61.0 \%)$ \\
\hline Total & $387(100 \%)$ & $387(100 \%)$ \\
\hline
\end{tabular}


Annals of International Medical and Dental Research

E-ISSN: 2395-2822 | P-ISSN: 2395-2814

Vol-8, Issue-1 | January-February 2022

DOI: 10.53339 /aimdr.2022.8.1.36

Page no- 274-286 | Section- Research Article (Obstetrics \& Gynaecology)

Table 9: Depression before \& after Delivery: Evaluated by DASS scale $(n=387)$

\begin{tabular}{|l|l|l|l|l|l|l|}
\hline $\begin{array}{l}\text { Depression } \\
\text { Before delivery }\end{array}$ & $\begin{array}{l}\text { Normal } \\
(\mathbf{\%})\end{array}$ & $\begin{array}{l}\text { Mild } \\
\mathbf{n}(\mathbf{\%})\end{array}$ & $\begin{array}{l}\text { Moderate } \\
\mathbf{n}(\mathbf{\%})\end{array}$ & $\begin{array}{l}\text { Severe } \\
\mathbf{n}(\mathbf{\%})\end{array}$ & $\begin{array}{l}\text { V severe } \\
\mathbf{n}(\%)\end{array}$ & $\begin{array}{l}\text { Total } \\
\mathbf{n}(\mathbf{\%})\end{array}$ \\
\hline Normal & $56(14.5)$ & $26(6.7)$ & $40(10.3)$ & $10(2.6)$ & $5(1.3)$ & $137(35.4)$ \\
\hline Mild & $25(6.5)$ & $12(3.1)$ & $10(2.6)$ & $4(1.0)$ & $5(1.3)$ & $569(14.5)$ \\
\hline Moderate & $26(6.7)$ & $14(3.6)$ & $45(11.6)$ & $26(6.7)$ & $6(1.6)$ & $117(30.2)$ \\
\hline Severe & $15(3.9)$ & $9(2.3)$ & $19(4.9)$ & $16(4.1)$ & $7(1.8)$ & $66(17.1)$ \\
\hline V.Severe & $3(0.8)$ & $1(0.3)$ & $5(1.3)$ & $2(0.5)$ & $0(0)$ & $11(2.8)$ \\
\hline Total & $125(32.3)$ & $62(16.0)$ & $119(30.7)$ & $58(15.0)$ & $23(5.9)$ & $387(100)$ \\
\hline
\end{tabular}

Table 10: Anxiety before \& after delivery: Evaluated by DASS scale $(\mathrm{n}=387)$

\begin{tabular}{|l|l|l|l|l|l|l|}
\hline $\begin{array}{l}\text { Anxiety } \\
\text { Before } \\
\text { delivery }\end{array}$ & Normal & Mild & Moderate & Severe & V.severe & Total \\
\cline { 2 - 7 } & $\mathbf{n}(\mathbf{0})$ & $\mathbf{n ~ ( \% )}$ & $\mathbf{n ~ ( \% )}$ & $\mathbf{n ~ ( \% )}$ & $\mathbf{n ~ ( \% )}$ & N (\%) \\
\hline Mild & $18(4.7)$ & $18(4.7)$ & $21(5.4)$ & $14(3.6)$ & $21(5.4)$ & $92(23.8)$ \\
\hline Moderate & $6(1.6)$ & $15(3.9)$ & $7(1.8)$ & $12(3.1)$ & $11(2.8)$ & $51(13.2)$ \\
\hline Severe & $5(1.3)$ & $10(2.6)$ & $9(2.3)$ & $13(3.4)$ & $14(3.6)$ & $51(13.2)$ \\
\hline V Severe & $8(2.1)$ & $22(5.7)$ & $14(3.6)$ & $15(3.9)$ & $46(11.9)$ & $105(27.1)$ \\
\hline Total & $62(16.0)$ & $83(21.4)$ & $69(17.8)$ & $71(18.3)$ & $102(26.4)$ & $387(100)$ \\
\hline
\end{tabular}

*McNemar Test was applied P value $=0.122$

Table 11: Stress before \& after delivery: Evaluated by DASS scale $(\mathrm{n}=387)$

\begin{tabular}{|c|c|c|c|c|c|c|}
\hline \multirow{2}{*}{$\begin{array}{l}\text { Stress } \\
\text { before } \\
\text { Delivery }\end{array}$} & \multicolumn{6}{|c|}{ Stress after delivery } \\
\hline & Normal & Mild & Moderate & Severe & V Severe & Total \\
\hline Normal & $154(39.8)$ & $32(8.3)$ & $44(11.4)$ & $4(1.0)$ & $0(0.0)$ & $234(60.5)$ \\
\hline Mild & $18(4.7)$ & $9(2.3)$ & $13(3.4)$ & $6(1.6)$ & $0(0.0)$ & $46(11.9)$ \\
\hline Moderate & $35(9.0)$ & $10(2.6)$ & $32(8.3)$ & $10(2.6)$ & $1(0.3)$ & $88(22.7)$ \\
\hline Severe & $9(2.3)$ & $1(0.3)$ & $7(1.8)$ & $1(0.3)$ & $0(0.0)$ & $18(4.7)$ \\
\hline V Severe & $1(0.3)$ & $0(0.0)$ & $0(0.0)$ & $0(0.0)$ & $0(0.0)$ & $1(0.3)$ \\
\hline Total & $217(56.1)$ & $52(13.4)$ & $96(24.8)$ & $21(5.4)$ & $1(0.3)$ & $387(100)$ \\
\hline
\end{tabular}

${ }^{*}$ McNemar Test was applied P value $=0.100$ 
Annals of International Medical and Dental Research E-ISSN: 2395-2822 | P-ISSN: 2395-2814

Vol-8, Issue-1 | January-February 2022

DOI: 10.53339 /aimdr.2022.8.1.36

Page no- 274-286 | Section- Research Article (Obstetrics \& Gynaecology)

Table 12: Presence of depression immediately after delivery and 4-6 weeks after delivery evaluated by EPDS scale $(\mathrm{n}=138)$.

\begin{tabular}{|l|l|l|l|l|}
\hline $\begin{array}{l}\text { Depression } \\
\text { Immediately } \\
\text { After deliver }\end{array}$ & Depression 4-6 weeks after delivery & p value \\
\cline { 2 - 5 } & Normal n (\%) & Possible n (\%) & Total n (\%) & \\
\hline Normal & $8(5.8)$ & $48(34.8)$ & $56(40.6)$ & $<0.001$ \\
\hline Possible & $15(10.9)$ & $67(48.6)$ & $82(59.4)$ & $*$ McNemar \\
\hline Total & $23(16.7)$ & $115(83.3)$ & $138(100)$ & Test was applied \\
\hline
\end{tabular}

Table 13: Presence of Depression before and after delivery using Depression Stress Anxiety Scale (DASS). (Derived from table 12)

\begin{tabular}{|c|c|c|c|c|c|c|}
\hline \multicolumn{3}{|c|}{$\begin{array}{l}\text { Depression Stress } \\
\text { Anxiety Scale (DASS) }\end{array}$} & \multicolumn{2}{|c|}{ Depression after delivery } & \multirow{2}{*}{\begin{tabular}{|l|} 
total \\
$310(80.1 \%)$ \\
\end{tabular}} & \multirow{2}{*}{$\begin{array}{l}\text { chi-square test } \\
\text { Mc Nemar test } \\
\text { For no=387 }\end{array}$} \\
\hline Depression & $\mathrm{NO}$ & Count (\%) & $\begin{array}{l}\text { No } \\
254(65.6 \%)\end{array}$ & $\begin{array}{l}\text { Yes } \\
56(14.5 \%)\end{array}$ & & \\
\hline $\begin{array}{l}\text { Before } \\
\text { delivery }\end{array}$ & YES & Count $(\%)$ & $52,13.4 \%$ & $25,6.5 \%$ & $77,19.9 \%$ & $\begin{array}{l}\text { Exact sig. } \\
\text { 2-sided,p } \\
\text { value773 }\end{array}$ \\
\hline Total & & Count $(\%)$ & $306(79.1 \%)$ & $81(20.9 \%)$ & $387(100 \%)$ & $\begin{array}{l}\text { Binomial } \\
\text { Distribution used }\end{array}$ \\
\hline
\end{tabular}

Table 14: Prevalence and Incidence of Antenatal and Postnatal Depression, Anxiety and Stress using DAS Scale.

\begin{tabular}{|l|l|l|l|}
\hline DASS (Depression Anxiety Stress) scale & Number(N/n) & Rate (\%) & $\mathbf{9 5 \%}$ CI \\
\hline Depression & $77 / 387$ & 19.9 & $15.9-23.8$ \\
\hline Ante partum Prevalence & $81 / 387$ & 20.9 & $16.8-24.9$ \\
\hline Postpartum Prevalence & $25 / 387$ & 6.45 & $4.0-8.9$ \\
\hline Incidence of depressionAnxiety & $156 / 387$ & 40.3 & $35.4-45.2$ \\
\hline Ante partum Prevalence & $173 / 387$ & 44.7 & $39.7-49.6$ \\
\hline Postpartum Prevalence & $88 / 387$ & 22.7 & $18.5-26.9$ \\
\hline Incidence of AnxietyStress & $5 / 387$ & 1.3 & $9.6-16.3$ \\
\hline Ante partum Prevalence & $5 / 387$ & 1.3 & $9.6-16.3$ \\
\hline Prevalence of Postpartum stress & $0 / 387$ & 0 & 0 \\
\hline Incidence of Stress & & & \\
\hline
\end{tabular}


Annals of International Medical and Dental Research E-ISSN: 2395-2822 | P-ISSN: 2395-2814

Vol-8, Issue-1 | January-February 2022

DOI: 10.53339 /aimdr.2022.8.1.36

Page no- 274-286 | Section- Research Article (Obstetrics \& Gynaecology)

Table 15: Association between antenatal depression (EPDS Scale) and certain selected factors

\begin{tabular}{|c|c|c|c|c|c|}
\hline \multirow[t]{2}{*}{ Risk factors } & \multicolumn{2}{|c|}{ Antenatal Depression EPDS } & \multirow[t]{2}{*}{ Total n(\%) } & \multirow[t]{2}{*}{ p value } & \multirow{2}{*}{$\begin{array}{ll}\text { OR } & (95 \% \\
\text { CI }) & \end{array}$} \\
\hline & Present n(\%) & Absent $n(\%)$ & & & \\
\hline \multicolumn{6}{|c|}{ A) Pregnancy Planning } \\
\hline Planned & $73(39.7)$ & $111(60.3)$ & $184(100.0)$ & \multirow[t]{2}{*}{0.223} & \\
\hline Unplanned & $93(45.8)$ & $110(54.2)$ & $203(100.0)$ & & \\
\hline \multicolumn{6}{|l|}{ B) Education } \\
\hline Up to High school & $80(47.1)$ & $90(52.9)$ & $170(100.0)$ & \multirow{4}{*}{0.195} & \multirow{4}{*}{1} \\
\hline Higher Secondary & $57(38.5)$ & $91(61.5)$ & $148(100.0)$ & & \\
\hline Graduate & $27(45.8)$ & $32(54.2)$ & $59(100.0)$ & & \\
\hline Postgraduate & $2(20.0)$ & $8(80.0)$ & $10(100.0)$ & & \\
\hline \multicolumn{6}{|l|}{ C)Occupation } \\
\hline Unskilled & $1(100.0)$ & $0(0.0)$ & $1(100.0)$ & \multirow{4}{*}{0.651} & \multirow{4}{*}{ NA } \\
\hline Semiskilled & $14(43.8)$ & $18(56.3)$ & $32(100.0)$ & & \\
\hline Professional & $5(35.7)$ & $9(64.3)$ & $14(100.0)$ & & \\
\hline Home maker & $146(42.9)$ & $194(57.1)$ & $340(100.0)$ & & \\
\hline \multicolumn{6}{|l|}{ D) Type of family } \\
\hline Nuclear & $112(38.2)$ & $181(61.8)$ & $293(100.0)$ & \multirow[b]{2}{*}{0.001} & \\
\hline Joint & $54(57.4)$ & $40(42.6)$ & $94(100.0)$ & & \\
\hline \multicolumn{6}{|c|}{ E) Marital Satisfaction (IMS) } \\
\hline Satisfied & $65(27.9)$ & $168(72.1)$ & $233(100.0$ & \multirow[b]{2}{*}{$<0.001$} & \multirow{2}{*}{$\begin{array}{l}0.5 \\
(0.2-1.1\end{array}$} \\
\hline Dissatisfied & 101(65.6) & $53(34.4)$ & $154(100.0)$ & & \\
\hline \multicolumn{6}{|c|}{ F) Relationship dissatisfaction (CMSS) } \\
\hline _ Present & $96(85.7)$ & $16(14.3)$ & $112(100.0)$ & \multirow[t]{2}{*}{$<0.001$} & \multirow{2}{*}{$\begin{array}{l}0.068 \\
(0.02-0.189)\end{array}$} \\
\hline Absent & $70(25.5)$ & $205(74.5)$ & $275(100.0)$ & & \\
\hline \multicolumn{6}{|c|}{ G) LES(LIFE EVENT SCALE) due to stress } \\
\hline $30 \%$ illness & $48(26.1)$ & 136(73.9) & $184(100.0)$ & \multirow{3}{*}{$<0.001$} & \multirow{3}{*}{$\begin{array}{l}1 \text { (Ref) } \\
0.87(0.39- \\
01 .(90) .0)\end{array}$} \\
\hline $50 \%$ illness & $93(52.8)$ & $83(47.2)$ & $176(100.0)$ & & \\
\hline $80 \%$ illness & $25(92.6)$ & $2(7.4)$ & $27(100.0)$ & & \\
\hline \multicolumn{6}{|c|}{ H) Sex of the previous child* } \\
\hline Female & $61(53.0)$ & $54(47.0)$ & 115(100.0) & \multirow{4}{*}{$<0.001$} & \multirow{4}{*}{ NA } \\
\hline Male and female & $4(100.0)$ & $0(0.0)$ & $4(100.0)$ & & \\
\hline Male and male & $0(0.0)$ & $4(100.0)$ & $4(100.0)$ & & \\
\hline Female and female & $11(91.7)$ & $1(8.3)$ & $12(100.0)$ & & \\
\hline
\end{tabular}


Annals of International Medical and Dental Research E-ISSN: 2395-2822 | P-ISSN: 2395-2814

Vol-8, Issue-1 | January-February 2022 DOI: 10.53339 /aimdr.2022.8.1.36

Page no- 274-286 | Section- Research Article (Obstetrics \& Gynaecology)

Table 16: marital dissatisfaction as measured byIMS scale and its association with depression

\begin{tabular}{|c|c|c|c|c|c|}
\hline \multirow[t]{2}{*}{ Risk factors } & \multicolumn{2}{|c|}{ Postnatal Depression } & \multirow[t]{2}{*}{ Total } & \multirow[t]{2}{*}{ p value } & \multirow[t]{2}{*}{ qr95\%CI } \\
\hline & Present $n(\%)$ & Absent( $\%)$ & & & \\
\hline \multicolumn{6}{|c|}{ I)Sex of the previous child* } \\
\hline Male & $24(20.7)$ & $92(79.3)$ & $116(100.0)$ & \multirow{5}{*}{0.612} & \multirow{5}{*}{ NA } \\
\hline Female & $27(23.5)$ & $88(76.5)$ & $115(100.0)$ & & \\
\hline Male and female & $0(0.0)$ & $4(100.0)$ & $4(100.0)$ & & \\
\hline Male and male & $0(0.0)$ & $4(100.0)$ & $4(100.0)$ & & \\
\hline Female and female & $2(16.7)$ & $10(83.3)$ & $12(100.0)$ & & \\
\hline \multicolumn{6}{|l|}{ J) Mode of delivery } \\
\hline SVD & $55(22.8)$ & $186(77.2)$ & $241(100.0)$ & \multirow{3}{*}{0.498} & $1.37(0.81-2.32)$ \\
\hline Assisted/ Instrumental & $1(20.0)$ & $4(80.0)$ & $5(100.0)$ & & $1.16(0.12-10.8)$ \\
\hline LSCS & $25(17.7)$ & $116(82.3)$ & $141(100.0)$ & & 1(Ref) \\
\hline \multicolumn{6}{|l|}{ K) Sex of the present baby } \\
\hline Male & $33(16.1)$ & $172(83.9)$ & $205(100.0)$ & \multirow[t]{2}{*}{0.013} & \multirow[t]{2}{*}{$1.86(1.13-3.09)$} \\
\hline Female & $48(26.4)$ & 134(73.6) & $182(100.0)$ & & \\
\hline \multicolumn{6}{|c|}{ L) Spontaneity of delivery\# } \\
\hline Spontaneous & $22(18.5)$ & $97(81.5)$ & $119(100.0)$ & \multirow[t]{2}{*}{0.190} & \multirow[t]{2}{*}{$1.5(0.79-3.08)$} \\
\hline Induced & $22(26.2)$ & $62(73.8)$ & $84(100.0)$ & & \\
\hline
\end{tabular}

${ }^{*} \mathrm{~N}=251$, only among multi para with live child; $\# \mathrm{~N}=203$, only among mothers with normaldelivery.

Table 17: relation between depression(EPDS)and certain related risk factors (EPDS Scale)

\begin{tabular}{|l|l|l|l|l}
\hline $\begin{array}{l}\text { Present } \\
\mathbf{n}(\%) \text { OR95\%CI }\end{array}$ & Absentn(\%) & Total n (\%) & p value & OR95\%CI \\
\hline
\end{tabular}

A) Sex of the previous child*

\begin{tabular}{|c|c|c|c|c|c|}
\hline Male & $42(36.2)$ & $74(63.8)$ & $116(100.0)$ & 0.034 & NA \\
\hline Female & $61(53.0)$ & $54(47.0)$ & $115(100.0)$ & & \\
\hline Male and female & $0(0.0)$ & $4(100.0)$ & $4(100.0)$ & & \\
\hline Male and male & $1(25.0)$ & $3(75.0)$ & $4(100.0)$ & & \\
\hline Female and female & $5(41.7)$ & $7(58.3)$ & $12(100.0)$ & & \\
\hline \multicolumn{6}{|l|}{ B) Mode of delivery } \\
\hline SVD & $91(37.8)$ & $150(62.2)$ & $241(100.0)$ & 0.498 & $1.18(0.77-1.8)$ \\
\hline $\begin{array}{l}\text { Assisted/ } \\
\text { Instrumental }\end{array}$ & $1(20.0)$ & $4(80.0)$ & $5(100.0)$ & & $\begin{array}{l}2.9(0.314- \\
26.4)\end{array}$ \\
\hline LSCS & $59(41.8)$ & $82(58.2)$ & 141(100.0) & & 1(Ref) \\
\hline \multicolumn{6}{|c|}{ C) Sex of the present baby } \\
\hline Male & $63(30.7)$ & $142(69.3)$ & 205(100.0) & \multirow[t]{2}{*}{$<0.001$} & \multirow[t]{2}{*}{ 2.1(1.39-3.2) } \\
\hline Female & $88(48.4)$ & $94(51.6)$ & 182(100.0) & & \\
\hline
\end{tabular}


Annals of International Medical and Dental Research

E-ISSN: 2395-2822 | P-ISSN: 2395-2814

Vol-8, Issue-1 | January-February 2022

DOI: $10.53339 /$ aimdr.2022.8.1.36

Page no- 274-286 | Section- Research Article (Obstetrics \& Gynaecology)

\begin{tabular}{|l|l|l|l|l|l|}
\hline D) Spontaneity of delivery\# \\
\hline Spontaneous & $42(35.3)$ & $77(64.7)$ & $119(100.0)$ & & $1.13(0.63-$ \\
\hline Induced & $32(38.1)$ & $52(61.9)$ & $84(100.0)$ & 0.683 & $2.02)$ \\
\hline
\end{tabular}

\section{DISCUSSION}

There were few and far studies in this region regarding mental health surveys during pregnancy, delivery and few months after delivery. Women admitted for delivery were screened for eligibility using inclusion and exclusion criteria.

From these participants. We studied various scales EPDS, DASS, CMSS, IMS, LES, LAS, We used the EPDS as a screening tool to identify women with depressive signs and DASS as a screening tool to identify Depression, Anxiety and Stress among these women. The prevalence of postpartum depression (PPD) was 39\% using EPDS. The present study, thus, strengthens the findings of previous studies and signifies the importance of identifying PPD, more so because none of these mothers had sought help for these symptoms, although

Their day to day functions impaired in high numbers with various shades of severity. The present study suggests that more than a third of the women had significantDepressive symptoms in the immediate postnatal period (within first 24-48 hours after delivery).[11,12,13] In the majority of such cases, the depressive symptoms persisted at six weeks follow-up. The strongest predictor of depressive symptoms at follow-up was the presence of depressive symptoms at baseline (i.e. prior to delivery).[14,15]

The rates were higher at 39\% than reported from other studies from India. One of the reasons could be that the present study assessed women in the immediate postpartum period still under the stress of labour.

Cut-off points: Cut off score is very essential for the screening purpose. At a cut off score there should be a balance between the sensitivity and specificity, so the test wouldn't lose its value either by having high false positive cases if the sensitivity is very high, or by having false negative cases if the specificity is very high. The recommended cut-off score for EDPS is 10. $16,17,18]$ Our finding is consistent with other studies ${ }^{\text {ee }}$ findings showing variability in the optimal cut-off scores between population studies. $[19,20,21]$

\section{CONCLUSIONS}

In conclusion this study highlights importance of screening for maternal mental health problems during Peri partum period. Along with Depression, we found higher prevalence of maternal Anxiety during Peripartum period which has to be given its due importance. Depression in immediate postpartum period is good predictor for increased risk of depression at 4-6 weeks postpartum. We found overlapping of depression, anxiety and stress symptoms in Peripartum period which need to be considered.

We found no statistically relevant differences in maternal sense of control during labour with mode of delivery. 
Annals of International Medical and Dental Research

E-ISSN: 2395-2822 | P-ISSN: 2395-2814

Vol-8, Issue-1 | January-February 2022

DOI: 10.53339 /aimdr.2022.8.1.36

Page no- 274-286 | Section- Research Article (Obstetrics \& Gynaecology)

\section{In Antenatal Period:}

- Marital dissatisfaction, Stressful life events, and joint family and previous both female children were significantly associated with depression.

- Whereas pregnancy planned / un planned, education and occupation of the mother was not associated with depression.

\section{In Immediate Post Partum Period:}

\section{REFERENCES}

1. Davé S, Petersen I, Sherr L, Nazareth I. Incidence of maternal and paternal depression in primary care: a cohort study using a primary care database. Arch Pediatr Adolesc Med. 2010;164(11):1038-44. doi: 10.1001/archpediatrics.2010.184.

2. Yonkers KA, Vigod S, Ross LE. Diagnosis, pathophysiology, and management of mood disorders in pregnant and postpartum women. Obstet Gynecol. 2011;117(4):961-977. doi: 10.1097/AOG.0b013e31821187a7.

3. Kakyo TA, Muliira JK, Mbalinda SN, Kizza IB, Muliira RS. Factors associated with depressive symptoms among postpartum mothers in a rural district in Uganda. Midwifery. 2012 Jun;28(3):374-9. doi: 10.1016/j.midw.2011.05.001. Epub 2011 May 23. PMID: 21601966.

4. Bener A, Gerber LM, Sheikh J. Prevalence of psychiatric disorders and associated risk factors in women during their postpartum period: a major public health problem and global comparison. Int J Womens Health. 2012;4:191-200. doi:10.2147/IJWH.S29380

5. Beck CT. A meta-analysis of predictors of postpartum depression. Nurs Res. 1996;45(5):297303. doi: 10.1097/00006199-199609000-00008.

6. Beck CT. Predictors of postpartum depression: an update. Nurs Res. 2001;50(5):275-85. doi: 10.1097/00006199-200109000-00004.

7. Wisner KL, Sit DK, McShea MC, et al. Onset timing, thoughts of self-harm, and diagnoses in postpartum women with screen-positive depression findings.
- Mode of delivery, planned/unplanned pregnancy, type of family, and induction of labour, maternal sense of control during labour, education and occupation of the mother did not have significant association with depression.

- Marital dissatisfaction, stressful life events and sex of the present baby being female were significantly associated with depression in immediate postpartum period.

JAMA Psychiatry. 2013;70(5):490-498. doi:10.1001/jamapsychiatry.2013.87

8. Bergink V, Lambregtse-van den Berg MP, Koorengevel KM, Kupka R, Kushner SA. First-onset psychosis occurring in the postpartum period: a prospective cohort study. J Clin Psychiatry. 2011;72(11):1531-7. doi: 10.4088/JCP.10m06648.

9. Patel V, Rahman A, Jacob KS, Hughes M. Effect of maternal mental health on infant growth in low income countries: new evidence from South Asia. BMJ. doi:10.1136/bmj.328.7443.820

10. Klier CM, Rosenblum KL, Zeller M, Steinhardt K, Bergemann N, Muzik M. A multirisk approach to predicting chronicity of postpartum depression symptoms. Depress Anxiety. 2008;25(8):718-24. doi: 10.1002/da.20419.

11. The American College of Obstetricians and Gynecologists Committee Opinion no. 630. Screening for perinatal depression. Obstet Gynecol. 2015;125(5):1268-1271. doi: 10.1097/01.AOG.0000465192.34779.dc.

12. Cox JL, Holden JM, Sagovsky R. Detection of postnatal depression. Development of the 10-item Edinburgh Postnatal Depression Scale. $\mathrm{Br} J$ Psychiatry. 1987;150:782-6. doi: 10.1192/bjp.150.6.782.

13. Nielsen Forman D, Videbech $\mathrm{P}$, Hedegaard $\mathrm{M}$, Dalby Salvig J, Secher NJ. Postpartum depression: identification of women at risk. BJOG. 2000;107(10):1210-7. doi: 10.1111/j.14710528.2000.tb11609.x. 
Annals of International Medical and Dental Research

E-ISSN: 2395-2822 | P-ISSN: 2395-2814

Vol-8, Issue-1 | January-February 2022

DOI: $10.53339 /$ aimdr.2022.8.1.36

Page no- 274-286 | Section- Research Article (Obstetrics \& Gynaecology)

14. Teissèdre F, Chabrol H. Detecting women at risk for postnatal depression using the Edinburgh Postnatal Depression Scale at 2 to 3 days postpartum. Can J Psychiatry. 2004;49(1):51-4. doi: 10.1177/070674370404900108.

15. Benjamin D, Chandramohan A, Annie IK, Prasad J, Jacob KS. Validation of the Tamil version of Edinburgh post-partum depression scale. J Obstet Gynecol India.2005;55:241-3.

16. Rwakarema M, Premji SS, Nyanza EC, Riziki P, Palacios-Derflingher L. Antenatal depression is associated with pregnancy-related anxiety, partner relations, and wealth in women in Northern Tanzania: a cross-sectional study. BMC Womens Health. 2015;15:68. doi:10.1186/s12905-015-0225-y

17. Gupta S, Kishore J, Mala YM, Ramji S, Aggarwal R. Postpartum depression in north Indian women: prevalence and risk factors. J Obstet Gynaecol India. 2013;63(4):223-229. doi:10.1007/s13224-013-0399-x

18. Adama ND, Foumane P, Olen JP, Dohbit JS, Meka EN, Mboudou E. Prevalence and Risk Factors of
Postpartum Depression in Yaounde, Cameroon. Open J Obstet Gynecol. 2015;5(11):608.

19. Fiala A, Švancara J, Klánová J, Kašpárek T. Sociodemographic and delivery risk Factors for developing postpartum depression in a sample of 3233 mothers from the Czech ELSPAC study. BMC Psych. 2017;17(1):104.

20. Ghosh A, Goswami S. Evaluation of post partum depression in a tertiary hospital. J Obstet Gynaecol India. 2011;61(5):528-530. doi:10.1007/s13224-0110077-9

21. Patel V, Rodrigues M, DeSouza N. Gender, poverty, and postnatal depression: a study of mothers in Goa, India. Am J Psychiatry. 2002;159(1):43-7. doi: 10.1176/appi.ajp.159.1.43.

Source of Support: Nil, Conflict of Interest: None declared 